\title{
Dynamics Analysis of an Epidemiological Model with Media Impact and Two Delays
}

\author{
Lianwen Wang, ${ }^{1}$ Yong $\mathrm{Li}^{2,3}$ and Liuyong Pang ${ }^{4}$ \\ ${ }^{1}$ School of Mathematics and Statistics, Central China Normal University, Wuhan 430079, China \\ ${ }^{2}$ School of Information and Mathematics, Yangtze University, Jingzhou 434023, China \\ ${ }^{3}$ Institute of Applied Mathematics, Yangtze University, Jingzhou 434023, China \\ ${ }^{4}$ Department of Mathematics, Huanghuai University, Zhumadian 463000, China \\ Correspondence should be addressed to Yong Li; gxbyl@163.com
}

Received 15 July 2015; Revised 7 December 2015; Accepted 21 December 2015

Academic Editor: Giovanni Garcea

Copyright (C) 2016 Lianwen Wang et al. This is an open access article distributed under the Creative Commons Attribution License, which permits unrestricted use, distribution, and reproduction in any medium, provided the original work is properly cited.

\begin{abstract}
This paper is concerned with exploring the global dynamics of SEIR epidemic model with media impact, which incorporates latency and relapse delays. The permanence of the model is carefully discussed. By suitable Lyapunov functionals, we establish the global stability of the equilibria. It is found that the basic reproduction number completely determines the threshold dynamics of the SEIR model. Finally, the impact of media on the epidemic spread is studied, which reveals that timely response of media and individuals may play a more key role in disease control.
\end{abstract}

\section{Introduction}

The investigation of dynamics of epidemiological models has been of importance in improving our understanding of disease control [1-6]. Media education has been an important control strategy for the emerging and reemerging epidemics, such as HIV/AIDS [7], SARS [2], Ebola virus disease (EVD), Middle East Respiratory Syndrome (MERS), which can not only alert the general public to the hazard from the infectious diseases but also educate the people about the requisite preventive measures such as wearing protective masks, vaccination, voluntary quarantine, and avoidance of congregated places. The extensive media education will bring about reducing the frequency and probability of potentially contagious contacts among the well-informed people [2-4]. In order to describe the impact of media on the diseases, Cui and his coauthors [3] used the transmission rate of the form $\beta \exp (-\alpha I)$ in SEI model with logistic growth, where $\beta$ is the transmission rate before media alert and $I$ denotes the number of infected individuals. This work provided a theoretical basis that a Hopf bifurcation can occur for weak media impact (small values of $\alpha$ ) while the model may have up to three endemic equilibria for strong media impact (large values of $\alpha$ ). Liu and Cui [4] proposed the transmission rate taking the form $\beta-\beta_{1} I /(m+I)$ to capture the impact of media on disease spread, where $\beta_{1} \leq \beta$ represents the reduced maximum value of the transmission rate when $I$ approaches infinite and $m$ reflects the reactive velocity of media coverage and individuals to the epidemic disease. For more details concerning the application of this transmission rate, we refer the reader to recent works $[5,6]$.

On the other hand, the individuals infected by infectious disease may develop symptoms after an incubation period [8], such as Hepatitis B virus (HBV), Hepatitis C virus (HCV), the human tuberculosis (TB), and Herpes simplex virus type 2 (HSV-2). The average latency period after the genital acquisition of HSV-2 is approximately 4 days [9], and latent tuberculosis may take months, years, or even decades to become infectious. Moreover, it has been found clinically that numerous diseases may make the recovered individuals suffer from a relapse of symptoms, including HBV [10], HCV [11], the majority of TB due to incomplete treatment [12], and genital HSV-2 [13, 14]. Recently, many epidemiological models incorporating both latency and relapse have been extensively investigated and many good results have been obtained (e.g., [15-19]). However, there are few investigation 
on both latency and relapse delays in the epidemiological models with media impact.

Suppose that the total population $N(t)$ at time $t$ is divided into four disjoint epidemic subclasses: susceptible $S(t)$, latent/exposed $E(t)$, infectious $I(t)$, and temporarily recovered $R(t)$, respectively. And $A$ denotes recruitment rate of susceptible class $S(t), \mu$ is natural death rate, $\nu$ indicates the death rate due to the disease, and $\eta$ represents the recovered rate for infectious class $I(t)$ due to natural recovery or treatment. As pointed by Cui et al. in [5], media can effectively reduce the contact rates among the population to a limited level. Hence, it may be more realistic to use the transmission rate $\beta-\beta_{1} I(t) /(m+I(t))$ compared to $\beta \exp (-m I(t))$. By incorporating media impact into the bilinear incidence rate, we now consider the incidence rate function as follows:

$$
f(S(t), I(t))=\left(\beta-\frac{\beta_{1} I(t)}{m+I(t)}\right) S(t) I(t) .
$$

In this work, we assume that the latency and relapse periods are constants, denoted by $\tau_{1}$ and $\tau_{2}$, respectively. Hence, the probabilities $\mathscr{P}_{1}(t)$ and $\mathscr{P}_{2}(t)$ of remaining in the latent class and the temporarily recovered class are the step-functions taking the forms

$$
\mathscr{P}_{i}(t)= \begin{cases}1, & 0 \leq t \leq \tau_{i}, \\ 0, & t>\tau_{i} .\end{cases}
$$

This suggests that all individuals remain in latent class for a constant period $\tau_{1}$ and in temporarily recovered class for a constant period $\tau_{2}$. One further assumes that the disease has been in the population for at least a time of $t>\tau:=$ $\max \left\{\tau_{1}, \tau_{2}\right\}$.

Following closely the ideas of $[5,8,15,18]$ and incorporating media impact, we consider the following integrodifferential epidemic model with latency and relapse delays:

$$
\begin{aligned}
\frac{d S(t)}{d t}= & A-\mu S(t)-f(S(t), I(t)), \\
E(t)= & \int_{t-\tau_{1}}^{t} e^{-\mu(t-r)} f(S(r), I(r)) d r, \\
\frac{d I(t)}{d t}= & e^{-\mu \tau_{1}} f\left(S\left(t-\tau_{1}\right), I\left(t-\tau_{1}\right)\right) \\
& -(\mu+\nu+\eta) I(t)+\eta e^{-\mu \tau_{2}} I\left(t-\tau_{2}\right), \\
R(t)= & \eta \int_{t-\tau_{2}}^{t} e^{-\mu(t-r)} I(r) d r .
\end{aligned}
$$

Differentiating the second and the fourth equations of (3), we derive the delay model

$$
\begin{aligned}
\frac{d S(t)}{d t}= & A-\mu S(t)-f(S(t), I(t)), \\
\frac{d E(t)}{d t}= & f(S(t), I(t))-\mu E(t) \\
& -e^{-\mu \tau_{1}} f\left(S\left(t-\tau_{1}\right), I\left(t-\tau_{1}\right)\right), \\
\frac{d I(t)}{d t}= & e^{-\mu \tau_{1}} f\left(S\left(t-\tau_{1}\right), I\left(t-\tau_{1}\right)\right) \\
& -(\mu+v+\eta) I(t)+\eta e^{-\mu \tau_{2}} I\left(t-\tau_{2}\right), \\
\frac{d R(t)}{d t}= & \eta I(t)-\mu R(t)-\eta e^{-\mu \tau_{2}} I\left(t-\tau_{2}\right),
\end{aligned}
$$

where the term $e^{-\mu \tau_{1}} f\left(S\left(t-\tau_{1}\right), I\left(t-\tau_{1}\right)\right)$ indicates the individuals surviving in the latent period $\tau_{1}$ and entering into infectious class at time $t$ and the term $\eta e^{-\mu \tau_{2}} I\left(t-\tau_{2}\right)$ represents the individuals surviving in temporarily recovered period $\tau_{2}$ and entering into infectious class at time $t$. The initial conditions for model (4) are given by

$$
\begin{aligned}
& S(\rho)=\varphi_{1}(\rho), \\
& E(\rho)=\varphi_{2}(\rho), \\
& I(\rho)=\varphi_{3}(\rho), \\
& R(\rho)=\varphi_{4}(\rho), \\
& \varphi_{i}(\rho) \geq 0, \quad \rho \in[-\tau, 0], \\
& \varphi_{i}(0)>0, \quad i=1,2,3,4 .
\end{aligned}
$$

Here, let $\varphi:=\left(\varphi_{1}, \varphi_{2}, \varphi_{3}, \varphi_{4}\right)^{T} \in C$, which denotes the Banach space $C\left([-\tau, 0], \mathbb{R}_{+0}^{4}\right)$ of continuous functions mapping the interval $[-\tau, 0]$ into $\mathbb{R}_{+0}^{4}$, equipped with the uniform norm defined by $\|\varphi\|=\sup _{\rho \in[-\tau, 0]}|\varphi|$, where $\mathbb{R}_{+0}^{4}=\left\{\left(x_{1}, x_{2}, x_{3}\right.\right.$, $\left.\left.x_{4}\right): x_{i} \geq 0, i=1,2,3,4\right\}$. In consideration of the continuity of the initial conditions, one requires

$$
\begin{aligned}
& E(0)=\int_{-\tau_{1}}^{0} e^{\mu \rho} f\left(\varphi_{1}(\rho), \varphi_{2}(\rho)\right) d \rho, \\
& R(0)=\eta \int_{-\tau_{2}}^{0} e^{\mu \rho} \varphi_{2}(\rho) d \rho .
\end{aligned}
$$

Our main aim of this study is concerned with investigating the global dynamics of model (4) and the impact of media on the disease spread. The basic structure of this paper is as follows. In the next section, we study the existence and the local stability of equilibria of (4). Section 3 carefully addresses the permanence of (4). In Section 4, global stability analysis of (4) is carried out. Finally, a discussion section ends this paper. 


\section{The Equilibria}

2.1. The Existence of Equilibria. Throughout this paper, denote $k:=\mu+\nu+\eta$.

Lemma 1. Any solution of model (4) with the initial conditions (5) and (6) is unique, positive, and bounded on $[0,+\infty)$. Moreover, the biologically feasible region

$$
\Omega=\left\{(S, E, I, R) \in \mathbb{R}_{+}^{4}: S+E+I+R \leq \frac{A}{\mu}\right\}
$$

is a positive invariant with respect to (4).

Proof. From the fundamental theory of functional differential equations [20], (4) admits a unique solution $(S(t), E(t)$, $I(t), R(t))^{T}$ satisfying the initial conditions (5) and (6).

Firstly, one shows that the solution $S(t)$ is positive, $\forall t \geq$ 0 . If not, we assign $t_{1}>0$ to be the first time such that $S\left(t_{1}\right)=$ 0 , which implies that $S(t)>0$ for $t \in\left[0, t_{1}\right)$. Thus, we must have $d S\left(t_{1}\right) / d t=A>0$ from the first equation of (4). Then there is a sufficiently small constant $\epsilon>0$ such that $S(t)<0$ holds for $t \in\left(t_{1}-\epsilon, t_{1}\right)$. This leads to a contradiction. So $S(t)>0$ for $t \geq 0$. Secondly, $I(t)>0$ also holds $\forall t \geq 0$. In fact, if $I(t)$ would lose its positivity and $t_{2}>0$ were the first time such that $I\left(t_{2}\right)=0$, then $I(t)>0$ for $t \in\left[0, t_{2}\right)$. Solving the third equation of $(4)$ on $\left[0, t_{2}\right]$ gives

$$
\begin{aligned}
& I\left(t_{2}\right)=e^{-k t_{2}}\{I(0) \\
& +\int_{0}^{t_{2}}\left[e^{-\mu \tau_{1}} f\left(S\left(r-\tau_{1}\right), I\left(r-\tau_{1}\right)\right)\right. \\
& \left.\left.+\eta e^{-\mu \tau_{2}} I\left(r-\tau_{2}\right)\right] e^{k r} d r\right\} .
\end{aligned}
$$

Due to $S(\rho), I(\rho)>0 \forall \rho \in\left[0, t_{2}\right)$, the right hand side of the above equality is positive, which yields that $I\left(t_{2}\right)>0$, contracting to $I\left(t_{2}\right)=0$. Thirdly, since the second equation of (4) is equivalent to the second equation of (3) and $S(t), I(t)>$ 0 , it follows from the second equation of (3) that $E(t)>0 \forall t \geq$ 0 . Similarly, we can obtain that $R(t)>0 \forall t \geq 0$. The positivity of solutions is proved.

Finally, the boundedness of the solutions is shown. Since $d N(t) / d t=A-\mu N(t)-\nu I(t) \leq A-\mu N(t)$, we get that $\limsup _{t \rightarrow \infty} N(t) \leq A / \mu$. This suggests that $S(t), E(t), I(t)$, $R(t)$ are bounded on $[0,+\infty)$. Hence, the feasible region $\Omega$ is a positive invariance that attracts all solutions of (4) in $\mathbb{R}_{+}^{4}$. The proof is completed.

Theorem 2. Model (4) admits a unique endemic equilibrium (EE) $E^{*}\left(S^{*}, E^{*}, I^{*}, R^{*}\right)$ if $\Re_{0}>1$, and there always exists a disease-free equilibrium (DFE) $E_{0}\left(S_{0}, 0,0,0\right)$.

Proof. Model (4) always has a disease-free equilibrium $E_{0}=$ $\left(S_{0}, 0,0,0\right)$, where $S_{0}=A / \mu$. Applying the theory of the next generation matrix in [21], we derive that

$$
\mathfrak{R}_{0}=\frac{\partial f\left(S_{0}, 0\right)}{\partial I} \frac{e^{-\mu \tau_{1}}}{k-\eta e^{-\mu \tau_{2}}}=\frac{e^{-\mu \tau_{1}} \beta A}{\mu\left(\mu+\nu+\eta-\eta e^{-\mu \tau_{2}}\right)}
$$

is the basic reproduction number of (4), which stands for the average number of new infections brought out by a typical infectious individual during the whole infectious period [22].

Let $(S, E, I, R)$ be any positive equilibrium (if it exists), then

$$
\begin{array}{r}
A-\mu S-f(S, I)=0, \\
f(S, I)-\mu E-e^{-\mu \tau_{1}} f(S, I)=0, \\
e^{-\mu \tau_{1}} f(S, I)-k I+\eta e^{-\mu \tau_{2}} I=0, \\
\eta I-\mu R-\eta e^{-\mu \tau_{2}} I=0 .
\end{array}
$$

Solving the second and the fourth equations of (10) yields that

$$
\begin{aligned}
& E=\frac{\left(1-e^{-\mu \tau_{1}}\right)}{\mu} f(S, I), \\
& R=\frac{\eta\left(1-e^{-\mu \tau_{2}}\right)}{\mu} I .
\end{aligned}
$$

From the first and the third equations of (10), eliminating $f(S, I)$ leads to

$$
S=\frac{1}{\mu}\left[A-e^{\mu \tau_{1}}\left(k-\eta e^{-\mu \tau_{2}}\right) I\right] .
$$

Denote $a=e^{\mu \tau_{1}}\left(k-\eta e^{-\mu \tau_{2}}\right)$. Because of $S=(A-a I) / \mu>0$, one has $I<A / a$. Now, one turns to studying the following equation:

$$
\phi(I):=f\left(\frac{A-a I}{\mu}, I\right)-a I=0, \quad I \in\left[0, \frac{A}{a}\right] .
$$

By $f(S, 0)=f(0, I)=0$, we get that $\phi(0)=0, \phi(A / a)=-A<$ 0 . Applying $\partial f\left(S_{0}, 0\right) / \partial S=0$ gives

$$
\begin{aligned}
\phi^{\prime}(0) & =-\frac{a}{\mu} \cdot \frac{\partial f\left(S_{0}, 0\right)}{\partial S}+\frac{\partial f\left(S_{0}, 0\right)}{\partial I}-a \\
& =a\left(\mathfrak{R}_{0}-1\right)>0,
\end{aligned}
$$

whence one obtains $\phi(I)>0$ when $\Re_{0}>1$ and $I$ is sufficiently small. One thus deduces that (13) admits a positive real root, denoted by $I^{*}$. This also suggests that model (4) at least admits positive equilibrium $E^{*}\left(S^{*}, E^{*}, I^{*}, R^{*}\right)$ from (11).

In fact, $E^{*}$ is proved to be a unique EE. From (13), it follows that $a=f\left(S^{*}, I^{*}\right) / I^{*}$. Due to $\beta \geq \beta_{1}$, we can examine that $\partial f\left(S^{*}, I^{*}\right) / \partial S>0$ and

$$
\begin{aligned}
\frac{\partial f\left(S^{*}, I^{*}\right)}{\partial I} & =\left(\beta-\frac{\beta_{1} I^{*}}{m+I^{*}} \frac{2 m+I^{*}}{m+I^{*}}\right) S^{*} \\
& <\left(\beta-\frac{\beta_{1} I^{*}}{m+I^{*}}\right) S^{*}=\frac{f\left(S^{*}, I^{*}\right)}{I^{*}},
\end{aligned}
$$

which leads to

$$
\begin{aligned}
\phi^{\prime}\left(I^{*}\right) & =-\frac{a}{\mu} \frac{\partial f\left(S^{*}, I^{*}\right)}{\partial S}+\frac{\partial f\left(S^{*}, I^{*}\right)}{\partial I}-a \\
& =-\frac{a}{\mu} \frac{\partial f\left(S^{*}, I^{*}\right)}{\partial S}+\frac{\partial f\left(S^{*}, I^{*}\right)}{\partial I}-\frac{f\left(S^{*}, I^{*}\right)}{I^{*}} \\
& <0,
\end{aligned}
$$


which implies that $\phi(I)$ strictly decreases at any positive equilibrium $E^{*}$. Note that $\phi(I)$ is continuously differentiable on $[0, A / a]$. Assume that (13) has more than one positive root; then there must exist certain one positive equilibrium $E^{+}\left(S^{+}, E^{+}, I^{+}, R^{+}\right)$such that $\phi^{\prime}\left(I^{+}\right) \geq 0$, resulting in a contraction. Thus, $I^{*}$ is unique and so is $E^{*}$ if $\mathfrak{R}_{0}>1$.

2.2. The Local Stability of Equilibria. In the following sections, one uses the notations $X=X(t)$ and $X_{\tau_{i}}=X\left(t-\tau_{i}\right), i=1,2$.

Theorem 3. For model (4), the DFE $E_{0}$ is locally asymptotically stable if $\Re_{0}<1$ but unstable if $\Re_{0}>1$. Moreover, the EE $E^{*}$ is locally asymptotically stable if $\Re_{0}>1$.

Proof. The characteristic equation of model (4) at some equilibrium is calculated as

$$
\left|\begin{array}{cccc}
-\left(\lambda+\mu+\frac{\partial f}{\partial S}\right) & 0 & -\frac{\partial f}{\partial I} & 0 \\
\left(1-e^{-(\lambda+\mu) \tau_{1}}\right) \frac{\partial f}{\partial S} & -(\lambda+\mu) & \left(1-e^{-(\lambda+\mu) \tau_{1}}\right) \frac{\partial f}{\partial I} & 0 \\
e^{-(\lambda+\mu) \tau_{1}} \frac{\partial f}{\partial S} & 0 & \pi & 0 \\
0 & 0 & \eta-\eta e^{-(\lambda+\mu) \tau_{2}} & -(\lambda+\mu)
\end{array}\right|
$$$$
=0 \text {, }
$$

where $\pi:=-\lambda-k+e^{-(\lambda+\mu) \tau_{1}} \partial f / \partial I+\eta e^{-(\lambda+\mu) \tau_{2}}$.

(1) By $\partial f\left(S_{0}, 0\right) / \partial S=0$, evaluating (17) at $E_{0}\left(S_{0}, 0,0,0\right)$ yields

$$
\begin{aligned}
& (\lambda+\mu)^{3}\left(\lambda+k-e^{-(\lambda+\mu) \tau_{1}} \frac{\partial f\left(S_{0}, 0\right)}{\partial I}-\eta e^{-(\lambda+\mu) \tau_{2}}\right) \\
& =0 .
\end{aligned}
$$

Clearly, an eigenvalue of (18) is $\lambda_{1}=-\mu<0$, and the remaining ones satisfy

$$
\psi(\lambda):=\lambda+k-e^{-(\lambda+\mu) \tau_{1}} \frac{\partial f\left(S_{0}, 0\right)}{\partial I}-\eta e^{-(\lambda+\mu) \tau_{2}}=0 .
$$

Suppose that $\mathfrak{R}_{0}>1$. From (19), we directly get

$$
\begin{aligned}
\psi(0)=k-e^{-\mu \tau_{1}} \frac{\partial f\left(S_{0}, 0\right)}{\partial I}-\eta e^{-\mu \tau_{2}}< & , \\
& \psi(+\infty)=+\infty,
\end{aligned}
$$

which means that (19) has at least one positive root. That is, $E_{0}$ is unstable if $\mathfrak{R}_{0}>1$. Suppose that $\mathfrak{R}_{0}<1$. Assign

$$
\Psi(\lambda):=\frac{\partial f\left(S_{0}, 0\right)}{\partial I} \frac{e^{-(\lambda+\mu) \tau_{1}}}{\lambda+k-\eta e^{-(\lambda+\mu) \tau_{2}}}
$$

Using (19) yields $\Psi(\lambda)=1$. Let $\lambda=x+y i(x, y \in \mathbb{R})$ be any root of (19). If $x \geq 0$, one has

$$
\begin{aligned}
|\Psi(\lambda)| & =\frac{\partial f\left(S_{0}, 0\right)}{\partial I} \frac{\left|e^{-(\lambda+\mu) \tau_{1}}\right|}{\left|\lambda+k-\eta e^{-(\lambda+\mu) \tau_{2}}\right|} \\
& \leq \frac{\partial f\left(S_{0}, 0\right)}{\partial I} \frac{e^{-\mu \tau_{1}}\left|e^{-x \tau_{1}}\right|}{\left|x+k-\eta e^{-\mu \tau_{2}} e^{-x \tau_{2}}\right|} \\
& \leq \frac{\partial f\left(S_{0}, 0\right)}{\partial I} \frac{e^{-\mu \tau_{1}}}{\left|k-\eta e^{-\mu \tau_{2}}\right|}=\mathfrak{R}_{0}<1,
\end{aligned}
$$

contradicting with (21), and thus $x<0$. So $E_{0}$ is locally asymptotically stable if $\boldsymbol{R}_{0}<1$.

(2) From (17), the characteristic equation of model (4) at $E^{*}\left(S^{*}, E^{*}, I^{*}, R^{*}\right)$ reads

$$
\begin{aligned}
& (\lambda+\mu)^{2}\left[\left(\lambda+\mu+\frac{\partial f\left(S^{*}, I^{*}\right)}{\partial S}\right)\right. \\
& \cdot\left(\lambda+k-\frac{\partial f\left(S^{*}, I^{*}\right)}{\partial I} e^{-(\lambda+\mu) \tau_{1}}-\eta e^{-(\lambda+\mu) \tau_{2}}\right) \\
& \left.+\frac{\partial f\left(S^{*}, I^{*}\right)}{\partial S} \frac{\partial f\left(S^{*}, I^{*}\right)}{\partial I} e^{-(\lambda+\mu) \tau_{1}}\right]=0 .
\end{aligned}
$$

Apparently, $\lambda_{1}=-\mu<0$ is an eigenvalue of (23). Furthermore, assume that (23) has another root $\lambda_{2}$ with $\operatorname{Re} \lambda_{2} \geq 0$, and then

$$
\begin{aligned}
\lambda_{2}+k= & \frac{\partial f\left(S^{*}, I^{*}\right)}{\partial I} \frac{\left(\lambda_{2}+\mu\right) e^{-\left(\lambda_{2}+\mu\right) \tau_{1}}}{\lambda_{2}+\mu+\partial f\left(S^{*}, I^{*}\right) / \partial S} \\
& +\eta e^{-\left(\lambda_{2}+\mu\right) \tau_{2}} .
\end{aligned}
$$

From (15) and $e^{-\mu \tau_{1}} f\left(S^{*}, I^{*}\right)+\eta e^{-\mu \tau_{2}} I^{*}=k I^{*}$, we can deduce that

$$
\begin{aligned}
& \left|\frac{\partial f\left(S^{*}, I^{*}\right)}{\partial I} \frac{\left(\lambda_{2}+\mu\right) e^{-\left(\lambda_{2}+\mu\right) \tau_{1}}}{\lambda_{2}+\mu+\partial f\left(S^{*}, I^{*}\right) / \partial S}+\eta e^{-\left(\lambda_{2}+\mu\right) \tau_{2}}\right| \\
& \leq\left|\frac{\partial f\left(S^{*}, I^{*}\right)}{\partial I} \frac{\left(\lambda_{2}+\mu\right) e^{-\left(\lambda_{2}+\mu\right) \tau_{1}}}{\lambda_{2}+\mu+\partial f\left(S^{*}, I^{*}\right) / \partial S}\right| \\
& \quad+\left|\eta e^{-\left(\lambda_{2}+\mu\right) \tau_{2}}\right|<e^{-\mu \tau_{1}} \frac{\partial f\left(S^{*}, I^{*}\right)}{\partial I}+\eta e^{-\mu \tau_{2}} \\
& \leq e^{-\mu \tau_{1}} \frac{f\left(S^{*}, I^{*}\right)}{I^{*}}+\eta e^{-\mu \tau_{2}}=k .
\end{aligned}
$$

Recall that the left side of (24) satisfies $\left|\lambda_{2}+k\right| \geq k$. This contradicts with (24). It follows from Theorem 9.17.4 in [23] that (23) does not admit any root with a nonnegative real part. So $E^{*}$ is locally asymptotically stable if $\mathfrak{R}_{0}>1$. This completes the proof.

\section{Permanence}

In order to study the permanence of model (4), we first discuss its uniform persistence when $\mathfrak{R}_{0}>1$ by the persistence theory for infinite dimensional systems [24]. 
Definition 4. Denote $\stackrel{\circ}{\Omega}$ as the interior of $\Omega$. If there is a constant $\varrho>0$ independent of initial values in $\stackrel{\circ}{\Omega}$, such that $\liminf _{t \rightarrow \infty} S \geq \varrho, \liminf _{t \rightarrow \infty} E \geq \varrho, \liminf _{t \rightarrow \infty} I \geq \varrho$, and $\lim _{\inf _{t \rightarrow \infty}} R \geq \varrho$, then model (4) is uniformly persistent in $\stackrel{\circ}{\Omega}$.

In the sequel, some notations and terminology are introduced. Denote $\Phi(t), t \geq 0$, as the family of solution operators with respect to (4). Consider $Y \in C$ with the uniform norm $\|\varphi\|$. Let us define the $\omega$-limit set as $\omega(y):=$ $\left\{z \in Y \quad \mid\right.$ there is a sequence $t_{n} \rightarrow \infty$ as $n \rightarrow$ $\infty$ with $\lim _{n \rightarrow \infty} \Phi\left(t_{n}\right) y=z$ \}. The semigroup $\Phi(t)$ is referred to as being asymptotically smooth, if for any bounded subset $\mathcal{U}$ of $Y$, for which $\Phi(t) \mathcal{U} \subset U \forall t \geq 0$, there is a compact set $\mathscr{A}$ such that $d(\Phi(t) \mathcal{U}, \mathscr{A}) \rightarrow 0$ as $t \rightarrow \infty$. Set

$$
\begin{aligned}
Y^{0} & =\left\{\varphi \in Y, \varphi_{i}(\rho)>0, \rho \in[-\tau, 0], i=2,3,4\right\}, \\
Y_{0} & =\left\{\varphi \in Y, \varphi_{2}(\rho)=0, \text { or } \varphi_{3}(\rho)=0, \text { or } \varphi_{4}(\rho)\right. \\
& =0, \rho \in[-\tau, 0]\} .
\end{aligned}
$$

It can be seen that $Y_{0}=Y / Y^{0}=\partial Y$, where $\partial Y$ represents the boundary of $Y$.

Lemma 5 (see [24], Theorem 4.2). Let the following conditions be satisfied:

(i) $Y^{0}$ is open and dense in $Y$ with $Y^{0} \cup Y_{0}=Y$ and $Y^{0} \cap$ $Y_{0}=\varnothing$.

(ii) The solution operators $\Phi(t)$ satisfy $\Phi(t): Y^{0} \rightarrow Y^{0}$, $\Phi(t): Y_{0} \rightarrow Y_{0}$.

(iii) $\Phi(t)$ is point dissipative in $Y$.

(iv) $\gamma^{+}(\mathcal{U})$ is bounded in $Y$ if $\mathscr{U}$ is bounded in $Y$.

(v) $\Phi(t)$ is asymptotically smooth.

(vi) $\mathscr{B}=\cup_{y \in \mathscr{B}_{b}} \omega(y)$ is isolated and has an acyclic covering $\mathscr{M}$, where $\mathscr{B}_{b}$ is the global attractor of $T(t)$ restricted to $Y_{0}$ and $\mathscr{M}=\cup_{i=1}^{j} \mathscr{M}_{i}, i=1,2, \ldots, j$.

(vii) $\forall \mathscr{M}_{i} \in \mathscr{M}, W^{s}\left(\mathscr{M}_{i}\right) \cap Y^{0}=\emptyset$ holds, where $W^{s}$ denotes the stable set.

Then $\Phi(t)$ is a uniform repeller with respect to $Y^{0}$; that $i$, there exists a constant $\varrho>0$ such that, $\forall y \in Y^{0}$, $\liminf _{t \rightarrow \infty} d\left(\Phi(t) y, Y_{0}\right) \geq \varrho$.

Theorem 6. Model (4) is permanent provided that $\mathfrak{R}_{0}>1$.

Proof. From (26), one examines that (i) and (ii) clearly hold. And (iii)-(iv) immediately follow from Lemma 1. It is seen that $\mathscr{B}=\left\{\widetilde{E}_{0}\right\}$ (here, $\left.\widetilde{E}_{0}:=\left(S_{0}, 0,0,0\right)\right)$ is isolated, which implies that the covering is simply $\mathscr{M}=\left\{\widetilde{E}_{0}\right\}$. Since no orbit connects $\widetilde{E}_{0}$ to itself in $Y_{0}$, we have that $\mathscr{M}$ is acyclic. Thus, (vi) is checked out.
Now, we prove that $W^{s}\left(\widetilde{E}_{0}\right) \cap Y^{0}=\emptyset$, where $W^{s}\left(\widetilde{E}_{0}\right)=$ $\left\{y \in Y^{0}: \lim _{t \rightarrow \infty} \Phi(t) y=\widetilde{E}_{0}\right\}$. Assume by contradiction that there is a solution $y=(S, E, I, R) \in W^{s}\left(\widetilde{E}_{0}\right) \cap Y^{0}$, such that

$$
\begin{aligned}
& \lim _{t \rightarrow \infty} S=S_{0}, \\
& \lim _{t \rightarrow \infty} E=0, \\
& \lim _{t \rightarrow \infty} I=0, \\
& \lim _{t \rightarrow \infty} R=0 .
\end{aligned}
$$

From Lemma 1 , we know that $S, I>0$ for $t \geq 0$. Choose a Lyapunov function

$$
\begin{aligned}
U(t)= & e^{\mu \tau_{1}} I+\int_{t-\tau_{1}}^{t} f(S(\sigma), I(\sigma)) d \sigma \\
& +\eta e^{\mu\left(\tau_{1}-\tau_{2}\right)} \int_{t-\tau_{2}}^{t} I(\sigma) d \sigma
\end{aligned}
$$

From (27), it follows that there exists $T_{0}>0$ such that $U\left(T_{0}\right)>$ 0 . And the time derivative of $U(t)$ along the solutions of (4) reads

$$
\begin{aligned}
\frac{d U(t)}{d t}= & e^{\mu \tau_{1}}\left[e^{-\mu \tau_{1}} f\left(S_{\tau_{1}}, I_{\tau_{1}}\right)-k I+\eta e^{-\mu \tau_{2}} I_{\tau_{2}}\right] \\
& +\left[f(S, I)-f\left(S_{\tau_{1}}, I_{\tau_{1}}\right)\right] \\
& +\eta e^{\mu\left(\tau_{1}-\tau_{2}\right)}\left(I-I_{\tau_{2}}\right) \\
= & f\left(S_{\tau_{1}}, I_{\tau_{1}}\right)-e^{\mu \tau_{1}} k I+\eta e^{\mu\left(\tau_{1}-\tau_{2}\right)} I_{\tau_{2}}+f(S, I) \\
& -f\left(S_{\tau_{1}}, I_{\tau_{1}}\right)+\eta e^{\mu\left(\tau_{1}-\tau_{2}\right)} I-\eta e^{\mu\left(\tau_{1}-\tau_{2}\right)} I_{\tau_{2}} \\
= & {\left[\frac{f(S, I)}{I}-e^{\mu \tau_{1}}\left(k-\eta e^{-\mu \tau_{2}}\right)\right] I . }
\end{aligned}
$$

By (27), we can use L'Hospital's rule, yielding

$$
\lim _{t \rightarrow \infty} \frac{f(S, I)}{I}=\frac{\partial f\left(S_{0}, 0\right)}{\partial I} .
$$

If $\mathfrak{R}_{0}>1$, by (9), then the function $U(t)$ is not decreasing when $t$ is large enough. For the given above $T_{0}$, one thus gets $U(t) \geq U\left(T_{0}\right)$ for $t \geq T_{0}$. Note that $U\left(T_{0}\right)>0$ prevents $(E, I, R)$ from converging to $(0,0,0)$ as $t \rightarrow \infty$, This contradicts to $S \rightarrow S_{0}$. For the dissipative system (4), uniform persistence is equivalent to permanence, completing the proof.

In an epidemiological sense, uniform persistence of model (4) implies that there are always infectious individuals if the disease is initially present and $\mathfrak{R}_{0}>1$.

\section{Global Stability}

We are now in a position to study the global asymptotic behaviors of model (4). 
Theorem 7. The DFE $E_{0}$ of model (4) is globally asymptotically stable in $\Omega$ if $\mathfrak{R}_{0} \leq 1$.

Proof. We still consider the Lyapunov function $U(t)$ defined in (29). Since $f\left(S_{0}, I\right) / I$ decreases with $I$ and $S \leq S_{0}$, applying L'Hospital's rule one obtains that

$$
\frac{f(S, I)}{I} \leq \frac{f\left(S_{0}, I\right)}{I} \leq \lim _{I \rightarrow 0^{+}} \frac{f\left(S_{0}, I\right)}{I}=\frac{\partial f\left(S_{0}, 0\right)}{\partial I}
$$

holds $\forall S, I>0$. From (29), the derivative of $U(t)$ along the solutions of (4) reads

$$
\begin{aligned}
\frac{d U(t)}{d t} & =\left[\frac{f(S, I)}{I}-e^{\mu \tau_{1}}\left(k-\eta e^{-\mu \tau_{2}}\right)\right] I \\
& \leq\left[\frac{\partial f\left(S_{0}, 0\right)}{\partial I}-e^{\mu \tau_{1}}\left(k-\eta e^{-\mu \tau_{2}}\right)\right] I \\
& =e^{\mu \tau_{1}}\left(k-\eta e^{-\mu \tau_{2}}\right)\left(\Re_{0}-1\right) I \leq 0
\end{aligned}
$$

if $\mathfrak{R}_{0} \leq 1$. Hence, when $\mathfrak{R}_{0} \leq 1$, there is a singleton $\left\{E_{0}\right\}$, as the maximal compact invariant set in $\{(S, E, I, R) \in$ $\Omega: d U(t) / d t=0\}$. Applying LaSalle's invariance principle immediately leads to the global asymptotic stability of $E_{0}$ in $\Omega$, completing the proof.

Let us introduce the well-known Volterra-type function, $\mathscr{H}(x)=x-1-\ln x, x>0$. Obviously, $\mathscr{H}(x)$ is positive-defined and reaches the global minimum at $x=1$ with $\mathscr{H}(1)=0$.

Theorem 8. The EE $E^{*}$ of model (4) is globally asymptotically stable in $\stackrel{\circ}{\Omega}$ if $\Re_{0}>1$.

Observe that the variables $E$ and $R$ in model (4) do not appear in the following subsystem:

$$
\begin{aligned}
& \frac{d S}{d t}=A-\mu S-f(S, I) \\
& \frac{d I}{d t}=e^{-\mu \tau_{1}} f\left(S_{\tau_{1}}, I_{\tau_{1}}\right)-k I+\eta e^{-\mu \tau_{2}} I_{\tau_{2}} .
\end{aligned}
$$

From Lemma 1 and Theorems 2, 3, and 6, one can derive the uniqueness, positivity, and boundedness of the solutions of (33). The region $\Pi=\left\{(S, I) \in \mathbb{R}_{+}^{2}: S+I \leq A / \mu\right\}$ is a positive invariant with (33) and denoting its interior by $\Pi^{\circ}$. Furthermore, (33) has a locally asymptotically stable trivial equilibrium $\varepsilon_{0}=\left(S_{0}, 0\right)$ if $\Re_{0}<1$, and it is unstable if $\mathfrak{R}_{0}>1$. There exists one unique locally asymptotically stable nontrivial equilibrium $\varepsilon^{*}\left(S^{*}, I^{*}\right)$ if $\mathfrak{R}_{0}>1$. Subsystem (33) is permanent when $\mathfrak{R}_{0}>1$. In order to prove Theorem 8 , we first explore the global stability of $\varepsilon^{*}$ of (33) based on its permanence.

Lemma 9. The equilibrium $\varepsilon^{*}$ of (33) is globally asymptotically stable in $\prod^{\circ}$ if $\mathfrak{R}_{0}>1$.
Proof. Construct the following Lyapunov function $V(t)=$ $W_{1}(t)+W_{2}(t)+W_{3}(t)$, where

$$
\begin{aligned}
& W_{1}(t)=\int_{S^{*}}^{S}\left(1-\frac{f\left(S^{*}, I^{*}\right)}{f\left(\sigma, I^{*}\right)}\right) d \sigma+e^{\mu \tau_{1}} I^{*} \mathscr{H}\left(\frac{I}{I^{*}}\right), \\
& W_{2}(t)=f\left(S^{*}, I^{*}\right) \int_{t-\tau_{1}}^{t} \mathscr{H}\left(\frac{f(S(\sigma), I(\sigma))}{f\left(S^{*}, I^{*}\right)}\right) d \sigma, \\
& W_{3}(t)=\eta e^{\mu\left(\tau_{1}-\tau_{2}\right)} I^{*} \int_{t-\tau_{2}}^{t} \mathscr{H}\left(\frac{I(\sigma)}{I^{*}}\right) d \sigma .
\end{aligned}
$$

It follows from Theorem 6 that the variables $S$ and $I$ are sufficiently bounded and bounded away from 0 . This ensures the boundedness of $V(t)$ for $t \geq 0$, and thus $V(t)$ is well defined. Together with the properties of $\mathscr{H}(x)$, we find that $V(t) \geq 0$ with global minimum 0 at $\varepsilon^{*}$. By $A=f\left(S^{*}, I^{*}\right)+\mu S^{*}$ and $k=e^{-\mu \tau_{1}} f\left(S^{*}, I^{*}\right) / I^{*}+\eta e^{-\mu \tau_{2}}$, differentiating $W_{1}(t)$ along the solutions of (33) yields

$$
\begin{aligned}
& \frac{d W_{1}(t)}{d t}=\left(1-\frac{f\left(S^{*}, I^{*}\right)}{f\left(S, I^{*}\right)}\right)\left[\mu S^{*}\left(1-\frac{S}{S^{*}}\right)\right. \\
& \left.+f\left(S^{*}, I^{*}\right)-f(S, I)\right]+\left(1-\frac{I^{*}}{I}\right)\left[f\left(S_{\tau_{1}}, I_{\tau_{1}}\right)\right. \\
& \left.-f\left(S^{*}, I^{*}\right) \frac{I}{I^{*}}-\eta e^{\mu\left(\tau_{1}-\tau_{2}\right)}\left(I-I_{\tau_{2}}\right)\right]=\mu S^{*}(1 \\
& \left.-\frac{S}{S^{*}}\right)\left(1-\frac{f\left(S^{*}, I^{*}\right)}{f\left(S, I^{*}\right)}\right)-f(S, I)+f\left(S^{*}, I^{*}\right)(1 \\
& \left.-\frac{f\left(S^{*}, I^{*}\right)}{f\left(S, I^{*}\right)}+\frac{f(S, I)}{f\left(S, I^{*}\right)}\right)+f\left(S_{\tau_{1}}, I_{\tau_{1}}\right) \\
& -f\left(S_{\tau_{1}}, I_{\tau_{1}}\right) \frac{I^{*}}{I}+f\left(S^{*}, I^{*}\right)\left(1-\frac{I}{I^{*}}\right) \\
& -\eta e^{\mu\left(\tau_{1}-\tau_{2}\right)}\left(I-I_{\tau_{2}}\right)+\eta e^{\mu\left(\tau_{1}-\tau_{2}\right)} I^{*}\left(1-\frac{I_{\tau_{2}}}{I}\right) .
\end{aligned}
$$

And the time derivatives of $W_{2}(t)$ and $W_{3}(t)$ along the solutions of (4), respectively, read

$$
\begin{aligned}
& \frac{d W_{2}(t)}{d t} \\
& =f\left(S^{*}, I^{*}\right)\left[\mathscr{H}\left(\frac{f(S, I)}{f\left(S^{*}, I^{*}\right)}\right)-\mathscr{H}\left(\frac{f\left(S_{\tau_{1}}, I_{\tau_{1}}\right)}{f\left(S^{*}, I^{*}\right)}\right)\right] \\
& =f(S, I)-f\left(S_{\tau_{1}}, I_{\tau_{1}}\right)+f\left(S^{*}, I^{*}\right) \ln \frac{f\left(S_{\tau_{1}}, I_{\tau_{1}}\right)}{f(S, I)}, \\
& \frac{d W_{3}(t)}{d t}=\eta e^{\mu\left(\tau_{1}-\tau_{2}\right)} I^{*}\left[\mathscr{H}\left(\frac{I}{I^{*}}\right)-\mathscr{H}\left(\frac{I_{\tau_{2}}}{I^{*}}\right)\right] \\
& =\eta e^{\mu\left(\tau_{1}-\tau_{2}\right)}\left(I-I_{\tau_{2}}\right)+\eta e^{\mu\left(\tau_{1}-\tau_{2}\right)} I^{*} \ln \frac{I_{\tau_{2}}}{I} .
\end{aligned}
$$


From (35)-(36), we therefore have

$$
\begin{aligned}
& \frac{d V(t)}{d t}=\frac{d W_{1}(t)}{d t}+\frac{d W_{2}(t)}{d t}+\frac{d W_{3}(t)}{d t} \\
& =\mu S^{*}\left(2-\frac{S}{S^{*}}-\frac{S^{*}}{S}\right) \\
& +\eta e^{\mu\left(\tau_{1}-\tau_{2}\right)} I^{*}\left(1-\frac{I_{\tau_{2}}}{I}+\ln \frac{I_{\tau_{2}}}{I}\right)+f\left(S^{*}, I^{*}\right) \\
& \cdot\left(1-\frac{f\left(S^{*}, I^{*}\right)}{f\left(S, I^{*}\right)}+\frac{f(S, I)}{f\left(S, I^{*}\right)}\right)+f\left(S^{*}, I^{*}\right) \\
& \cdot\left(1-\frac{I}{I^{*}}-\frac{I^{*} f\left(S_{\tau_{1}}, I_{\tau_{1}}\right)}{I f\left(S^{*}, I^{*}\right)}+\ln \frac{f\left(S_{\tau_{1}}, I_{\tau_{1}}\right)}{f(S, I)}\right) .
\end{aligned}
$$

Note that

$$
\begin{aligned}
\ln \frac{f\left(S_{\tau_{1}}, I_{\tau_{1}}\right)}{f(S, I)}= & \ln \frac{f\left(S^{*}, I^{*}\right)}{f\left(S, I^{*}\right)}+\ln \frac{I^{*} f\left(S_{\tau_{1}}, I_{\tau_{1}}\right)}{I f\left(S^{*}, I^{*}\right)} \\
& +\ln \frac{I f\left(S, I^{*}\right)}{I^{*} f(S, I)} .
\end{aligned}
$$

Further, it can be found that

$$
\begin{aligned}
& \frac{d V(t)}{d t}=\mu S^{*}\left(2-\frac{S}{S^{*}}-\frac{S^{*}}{S}\right)-\eta e^{\mu\left(\tau_{1}-\tau_{2}\right)} I^{*} \mathscr{H}\left(\frac{I_{\tau_{2}}}{I}\right) \\
& +f\left(S^{*}, I^{*}\right)\left(1-\frac{f\left(S^{*}, I^{*}\right)}{f\left(S, I^{*}\right)}+\ln \frac{f\left(S^{*}, I^{*}\right)}{f\left(S, I^{*}\right)}\right) \\
& +f\left(S^{*}, I^{*}\right)\left(1-\frac{I^{*} f\left(S_{\tau_{1}}, I_{\tau_{1}}\right)}{I f\left(S^{*}, I^{*}\right)}\right. \\
& \left.+\ln \frac{I^{*} f\left(S_{\tau_{1}}, I_{\tau_{1}}\right)}{I f\left(S^{*}, I^{*}\right)}\right)+f\left(S^{*}, I^{*}\right)\left(1-\frac{I f\left(S, I^{*}\right)}{I^{*} f(S, I)}\right. \\
& \left.+\ln \frac{I f\left(S, I^{*}\right)}{I^{*} f(S, I)}\right)+f\left(S^{*}, I^{*}\right)\left(\frac{f(S, I)}{f\left(S, I^{*}\right)}-\frac{I}{I^{*}}\right. \\
& \left.+\frac{I f\left(S, I^{*}\right)}{I^{*} f(S, I)}-1\right)=\mu S^{*}\left(2-\frac{S}{S^{*}}-\frac{S^{*}}{S}\right) \\
& -\eta e^{\mu\left(\tau_{1}-\tau_{2}\right)} I^{*} \mathscr{H}\left(\frac{I_{\tau_{2}}}{I}\right)-f\left(S^{*}, I^{*}\right)\left[\mathscr{H}\left(\frac{S^{*}}{S}\right)\right. \\
& \left.+\mathscr{H}\left(\frac{I^{*} f\left(S_{\tau_{1}}, I_{\tau_{1}}\right)}{I f\left(S^{*}, I^{*}\right)}\right)+\mathscr{H}\left(\frac{I f\left(S, I^{*}\right)}{I^{*} f(S, I)}\right)\right] \\
& +f\left(S^{*}, I^{*}\right)\left(1-\frac{f\left(S, I^{*}\right)}{f(S, I)}\right)\left(\frac{f(S, I)}{f\left(S, I^{*}\right)}-\frac{I}{I^{*}}\right) .
\end{aligned}
$$

We can show that $f(S, I)$ is nondecreasing and concave down with respect to $I$ since

$$
\begin{aligned}
\frac{\partial f(S, I)}{\partial I} & =\left(\beta-\beta_{1} \frac{2 m I+I^{2}}{(m+I)^{2}}\right) S \geq 0, \\
\frac{\partial^{2} f(S, I)}{\partial I^{2}} & =-\frac{2 \beta_{1} m^{2} S}{(m+I)^{3}} \leq 0,
\end{aligned}
$$

which ensure that

$$
\left(1-\frac{f\left(S, I^{*}\right)}{f(S, I)}\right)\left(\frac{f(S, I)}{f\left(S, I^{*}\right)}-\frac{I}{I^{*}}\right) \leq 0 .
$$

From the properties of $\mathscr{H}(x)$, one deduces that $d V(t) / d t \leq 0$, and the strict equality holds if and only if $S=S^{*}, I=$ $I^{*}$, and $S_{\tau_{1}}=S^{*}, I_{\tau_{1}}=I^{*}$. It follows from Theorem 5.3.1 in [25] that the solutions of system (33) converge to $\omega$, the maximal invariant set in $\{d V(t) / d t=0\}$. Accordingly, we obtain $\Phi=\left(S^{*}, I^{*}\right)$. LaSalle's invariance principle for delay differential systems (see, e.g., [26]) indicates that $\varepsilon^{*}$ is globally asymptotically stable in $\prod^{\circ}$. The proof is completed.

Proof of Theorem 8. Let $(S, E, I, R)$ be a positive solution of model (4) with initial conditions (5) and (6). Applying Lemma 9, one derives that $S, S_{\tau_{1}} \rightarrow S^{*}$ and $I, I_{\tau_{1}} \rightarrow I^{*}$, as $t \rightarrow+\infty$ if $\mathfrak{R}_{0}>1$. By L'Hospital's rule, it follows from the second and the fourth equations of (3) that

$$
\begin{aligned}
\lim _{t \rightarrow+\infty} E & =\lim _{t \rightarrow+\infty} \int_{t-\tau_{1}}^{t} e^{-\mu(t-s)} f(S(s), I(s)) d s \\
& =\frac{1}{\mu} \lim _{t \rightarrow+\infty}\left(f(S, I)-e^{-\mu \tau_{1}} f\left(S_{\tau_{1}}, I_{\tau_{1}}\right)\right) \\
& =\frac{\left(1-e^{-\mu \tau_{1}}\right)}{\mu} f\left(S^{*}, I^{*}\right)=E^{*}, \\
\lim _{t \rightarrow+\infty} R & =\lim _{t \rightarrow+\infty} \eta \int_{t-\tau_{2}}^{t} e^{-\mu(t-s)} I(s) d s \\
& =\frac{\eta}{\mu} \lim _{t \rightarrow+\infty}\left(I-e^{-\mu \tau_{2}} I_{\tau_{2}}\right)=\frac{\eta\left(1-e^{-\mu \tau_{2}}\right)}{\mu} I^{*} \\
& =R^{*} .
\end{aligned}
$$

The fact that $E^{*}$ is local stable when it exists, implies that it is also globally asymptotically stable in $\Omega^{\circ}$ if $\Re_{0}>1$. We finish the proof.

\section{Concluding Remarks}

In this paper, we propose SEIR epidemic model with media impact, which incorporates latent and relapse delays. One focuses on analyzing the permanence and global stability of model (4). In detail, we show that (4) is permanent when $\mathfrak{R}_{0}>1$. Based on the permanence obtained, we carry out global stability analysis of the equilibria by proper Lyapunov functionals. It is found that (4) is still a threshold dynamical system. 


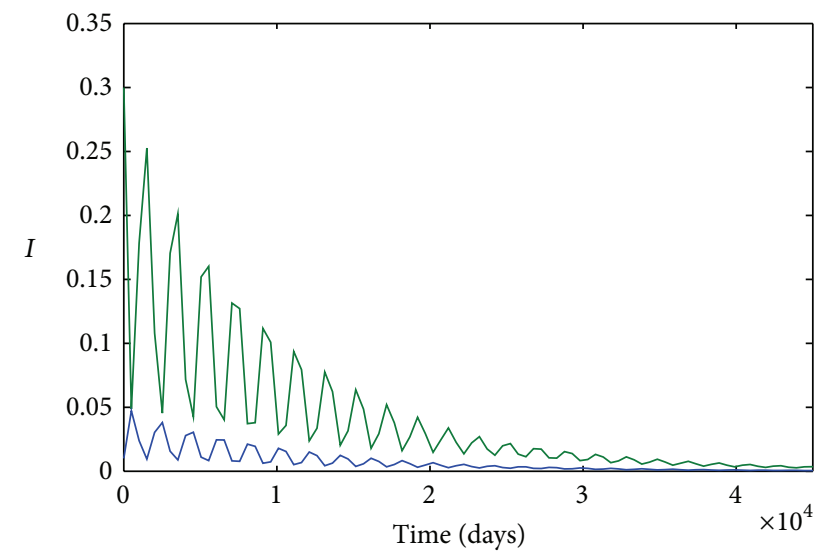

FIGURE 1: The DFE $E_{0}$ of model (4) is globally asymptotically stable for different initiate conditions when $\mathfrak{R}_{0}=0.0077$, where we take $\left(A, \mu, \eta, \tau_{1}, \tau_{2}, \beta, \beta_{1}, m\right)=(1 / 7300,1 / 7300,0,3.5,4$, $27,0.0001,0.00005,10)$.

Now several numerical simulations are demonstrated to check Theorems 7 and 8 . For the purpose of controlling the spread of genital HSV-2, for instance, we implement media propaganda and education. Choose $A=\mu=1 / 7300, \nu=0$, $\eta=3.5$, and $\tau_{2}=27$, as used in Blower et al. [27]. Since the average incubation period after the genital acquisition of HSV-2 is approximately 4 days (range, 2 to 12 days) [9], we may take $\tau_{1}=4$. When $\beta=0.0001, \beta_{1}=0.00005$, and $m=10$, we then get that $\Re_{0}=0.0077$. Figure 1 shows us that the disease-free equilibrium $E_{0}$ of (4) is globally asymptotically stable for two different initiate conditions $(S(0), E(0), I(0), R(0))=(0.69,0.05,0.3,0.05)$ (green line) and $(S(0), E(0), I(0), R(0))=(0.8,0.05,0.01,0.05)$ (blue line). And, given that $\beta=0.02, \beta_{1}=0.01$, and $m=10$, direct calculation gives $\Re_{0}=1.5308$. From Theorem 8 one knows that the unique endemic equilibrium $E^{*}$ is globally asymptotically stable as shown in Figure 2 for the different initiate conditions used in Figure 1. We observe that there is no sustained oscillatory solution and thus media control and the two delays do not have fundamental influence on the qualitative behaviors of model (4).

Additionally, since $d \Re_{0} / d \tau_{1}<0$ and $d \Re_{0} / d \tau_{2}<0$, it is an advantage for controlling the disease spread to increase both latent and relapse delays. In practice, the latent period may be hard to change, but the likelihood of symptomatic recurrence [14] and the frequency of subclinical (asymptomatic) viral shedding [13] can be substantially reduced under suppressive therapy rather than episodic treatment, such that the relapse period (delay) can be lengthened.

Note that media education does not change the basic reproduction number $\mathfrak{R}_{0}$ [4]. However, the greater the reactive velocity of media coverage and individuals (i.e., the smaller the value of $m$ ), the endemic level $I^{*}$ will be controlled to a much lower level, seeing the blue lines shown in Figure 3 (where we change the values of $m$ and $\beta_{1}$ but keep the same initiate condition $(S(0), E(0), I(0), R(0))=$ $(0.8,0.05,0.01,0.05)$ and the reminding parameters values are the same with Figure 2). On the contrary, if media

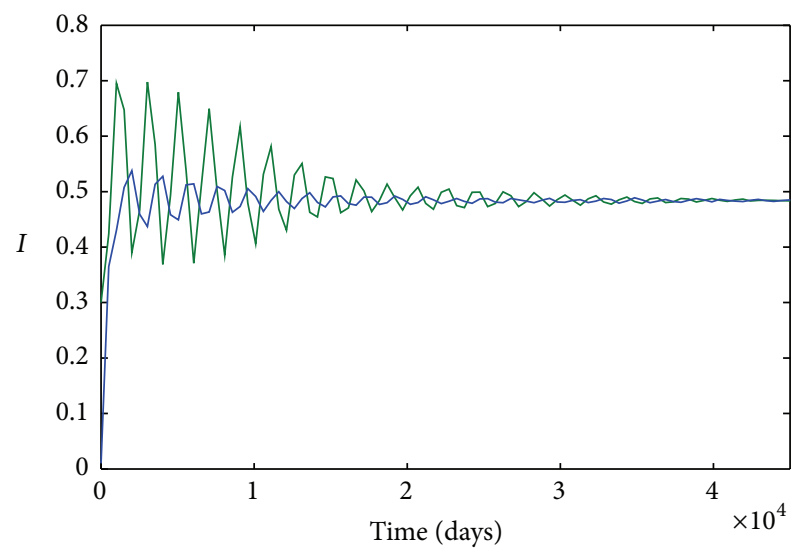

Figure 2: The EE $E^{*}$ of model (4) is globally asymptotically stable when $\mathfrak{R}_{0}=1.5308$, where $\beta=0.02, \beta_{1}=0.01$, and initiate conditions and the reminding parameters values are kept the same as Figure 1 .

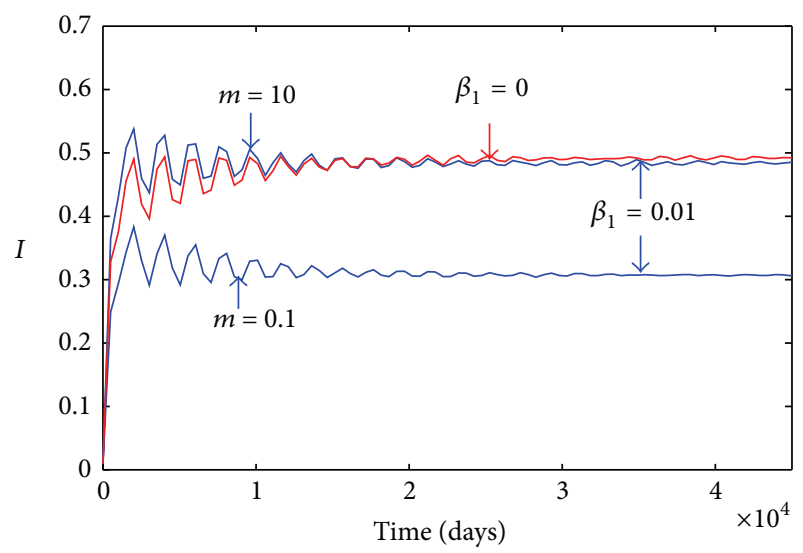

FIGURE 3: The impact of media on the genital HSV-2 under the same initiate condition $(S(0), E(0), I(0), R(0))=(0.8,0.05,0.01,0.05)$ except for the values of $m$ and $\beta_{1}$.

departments and the public do not respond timely to the epidemic, the effect of media propaganda on the disease transmission is almost the same with the case with no media impact (i.e., $\beta_{1}=0$, seeing the red line in Figure 3). Hence, timely response of media coverage and individuals plays a more key role in controlling the epidemic.

\section{Conflict of Interests}

The authors declare that there is no conflict of interests regarding the publishing of this paper.

\section{Acknowledgments}

The work was partially supported by the Open Research Fund Program of Institute of Applied Mathematics Yangtze University (no. KF1507), Basic Subject of Scientific Research and Development Fund of Yangtze University (no. 2014JCY001), 
and Key Scientic Research Project of Higher Education Institutions of Henan Province (16A110005).

\section{References}

[1] W. O. Kermack and A. G. McKendrick, "A contribution to the mathematical theory of epidemics," Proceedings of the Royal Society of London, Series A: Mathematical, Physical and Engineering Sciences, vol. 115, no. 772, pp. 700-721, 1927.

[2] R. S. Liu, J. H. Wu, and H. P. Zhu, "Media/psychological impact on multiple outbreaks of emerging infectious diseases," Computational and Mathematical Methods in Medicine, vol. 8, no. 3, pp. 153-164, 2007.

[3] J. A. Cui, Y. H. Sun, and H. P. Zhu, "The impact of media on the control of infectious diseases," Journal of Dynamics and Differential Equations, vol. 20, no. 1, pp. 31-53, 2008.

[4] Y. Liu and J.-A. Cui, "The impact of media coverage on the dynamics of infectious disease," International Journal of Biomathematics, vol. 1, no. 1, pp. 65-74, 2008.

[5] J.-A. Cui, X. Tao, and H. P. Zhu, "An SIS infection model incorporating media coverage," The Rocky Mountain Journal of Mathematics, vol. 38, no. 5, pp. 1323-1334, 2008.

[6] J. M. Tchuenche, N. Dube, C. P. Bhunu, R. J. Smith, and C. T. Bauch, "The impact of media coverage on the transmission dynamics of human influenza," BMC Public Health, vol. 11, supplement 1, article S5, 2011.

[7] S. Broder and R. C. Gallo, "A pathogenic retrovirus (HTLV-III) linked to AIDS," The New England Journal of Medicine, vol. 311, no. 20, pp. 1292-1297, 1984.

[8] P. van den Driessche, L. Wang, and X. F. Zou, "Modeling diseases with latency and relapse," Mathematical Biosciences and Engineering, vol. 4, no. 2, pp. 205-219, 2007.

[9] D. W. Kimberlin and D. J. Rouse, “Genital herpes," The New England Journal of Medicine, vol. 350, no. 19, pp. 1970-1977, 2004.

[10] A. Marzano, S. Gaia, V. Ghisetti et al., "Viral load at the time of liver transplantation and risk of hepatitis B virus recurrence," Liver Transplantation, vol. 11, no. 4, pp. 402-409, 2005.

[11] A. Y. Kim, J. Schulze zur Wiesch, T. Kuntzen et al., "Impaired hepatitis $\mathrm{C}$ virus-specific $\mathrm{T}$ cell responses and recurrent hepatitis C virus in HIV coinfection," PLoS Medicine, vol. 3, no. 12, article e492, 2006.

[12] M.-L. Lambert, E. Hasker, A. Van Deun, D. Roberfroid, M. Boelaert, and P. Van der Stuyft, "Recurrence in tuberculosis: relapse or reinfection?" The Lancet Infectious Diseases, vol. 3, no. 5, pp. 282-287, 2003.

[13] A. Wald, J. Zeh, G. Barnum, L. G. Davis, and L. Corey, "Suppression of subclinical shedding of herpes simplex virus type 2 with acyclovir," Annals of Internal Medicine, vol. 124, no. 1, part 1, pp. 8-15, 1996.

[14] M. Reitano, S. Tyring, W. Lang et al., "Valaciclovir for the suppression of recurrent genital herpes simplex virus infection: a large-scale dose range-finding study," Journal of Infectious Diseases, vol. 178, no. 3, pp. 603-610, 1998.

[15] P. van den Driessche and X. Zou, "Modeling relapse in infectious diseases," Mathematical Biosciences, vol. 207, no. 1, pp. 89103, 2007.

[16] K. L. Cooke and P. van den Driessche, "Analysis of an SEIRS epidemic model with two delays," Journal of Mathematical Biology, vol. 35, no. 2, pp. 240-260, 1996.
[17] S. Q. Liu, S. K. Wang, and L. Wang, "Global dynamics of delay epidemic models with nonlinear incidence rate and relapse," Nonlinear Analysis. Real World Applications, vol. 12, no. 1, pp. 119-127, 2011.

[18] R. Xu, "Global dynamics of an SEIRI epidemiological model with time delay," Applied Mathematics and Computation, vol. 232, pp. 436-444, 2014.

[19] L. L. Liu, J. Wang, and X. Liu, "Global stability of an SEIR epidemic model with age-dependent latency and relapse," Nonlinear Analysis: Real World Applications, vol. 24, pp. 18-35, 2015.

[20] J. Hale, Theory of Functional Differential Equations, Springer, Heidelberg, Germany, 1977.

[21] P. van den Driessche and J. Watmough, "Reproduction numbers and sub-threshold endemic equilibria for compartmental models of disease transmission," Mathematical Biosciences, vol. 180, no. 1, pp. 29-48, 2002.

[22] R. A. Ross, The Prevention of Malaria, John Murray, London, UK, 2nd edition, 1911.

[23] L. E. El'sgol'ts and S. B. Norkin, Introduction to the Theory and Application of Differential Equations with Deviating Arguments, Academic Press, New York, NY, USA, 1973.

[24] J. K. Hale and P. Waltman, "Persistence in infinite-dimensional systems," SIAM Journal on Mathematical Analysis, vol. 20, no. 2, pp. 388-395, 1989.

[25] J. K. Hale and S. M. Verduyn Lunel, Introduction to Functional Differential Equations, Springer, New York, NY, USA, 1993.

[26] J. R. Haddock and J. Terjéki, "Liapunov-Razumikhin functions and an invariance principle for functional-differential equations," Journal of Differential Equations, vol. 48, no. 1, pp. 95-122, 1983.

[27] S. M. Blower, T. C. Porco, and G. Darby, "Predicting and preventing the emergence of antiviral drug resistance in HSV2," Nature Medicine, vol. 4, no. 6, pp. 673-678, 1998. 


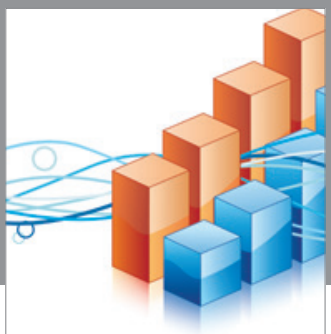

Advances in

Operations Research

vatem alat4

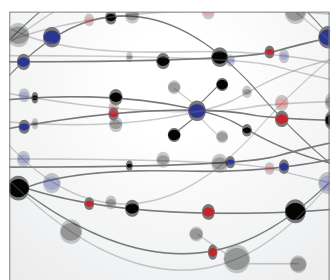

\section{The Scientific} World Journal
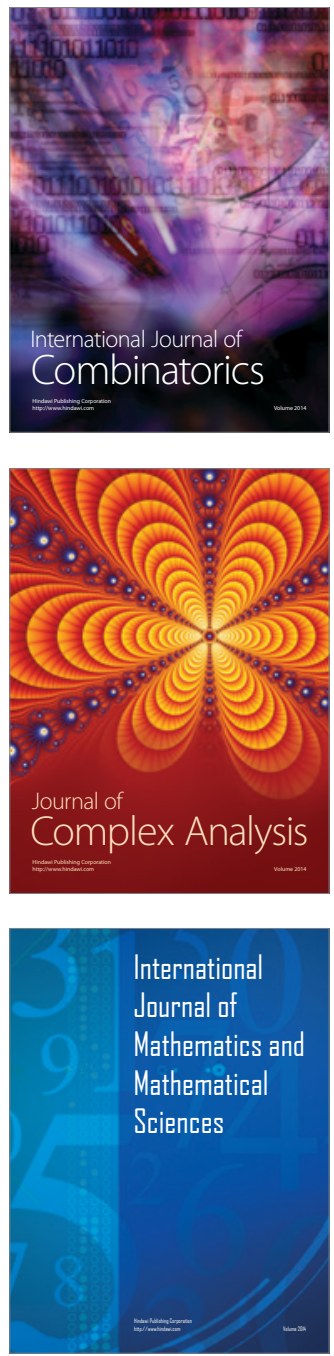
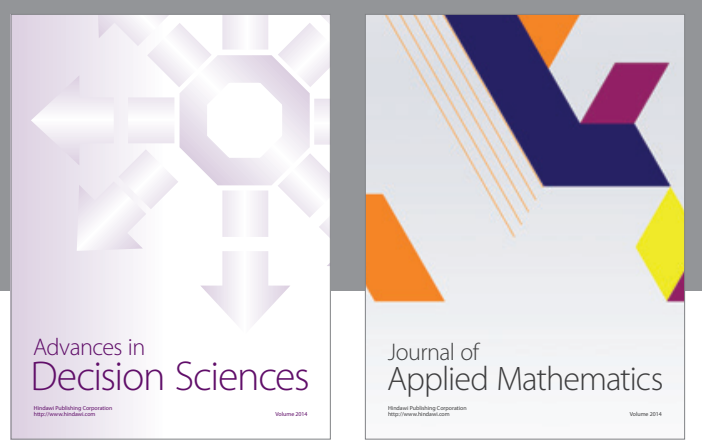

Algebra

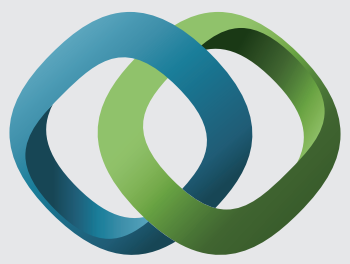

\section{Hindawi}

Submit your manuscripts at

http://www.hindawi.com
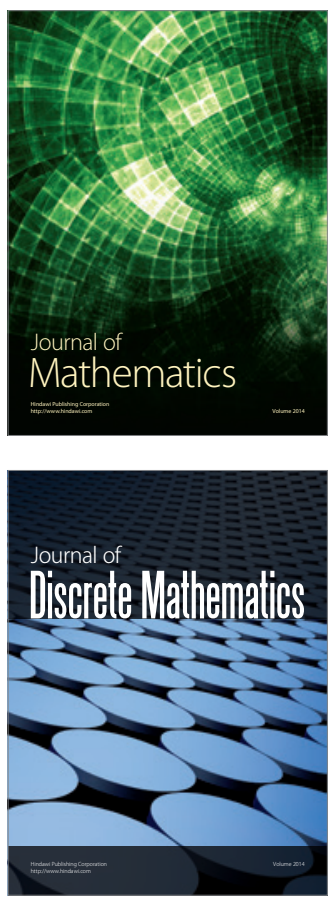

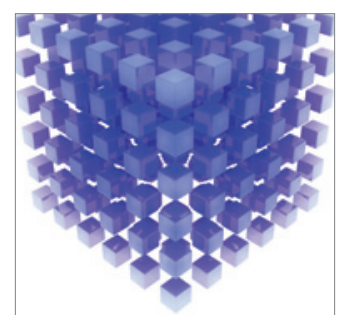

Mathematical Problems in Engineering
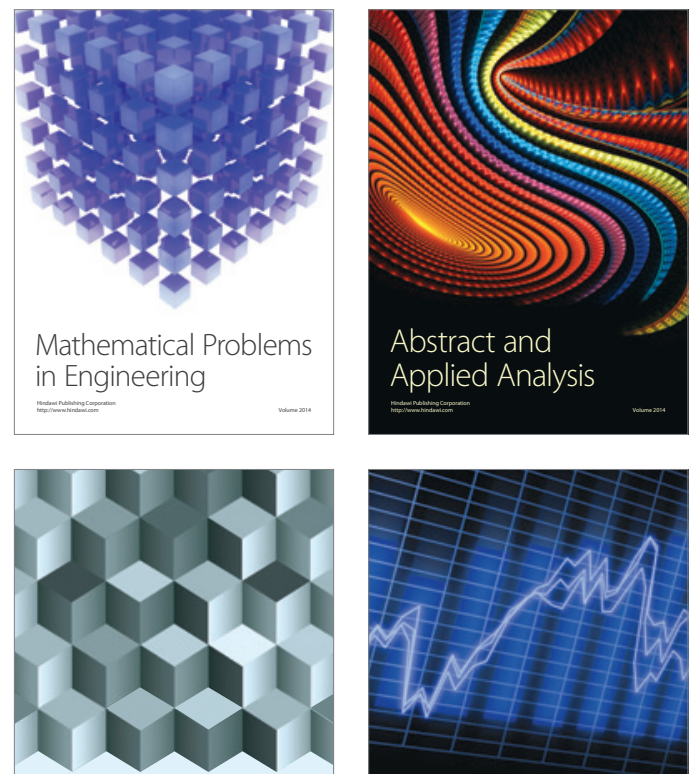

Journal of

Function Spaces

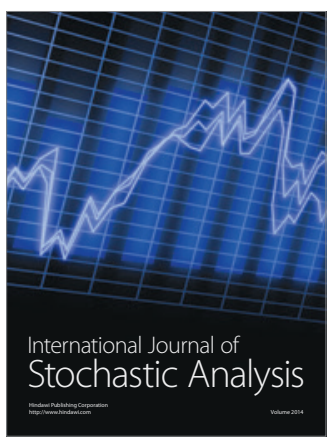

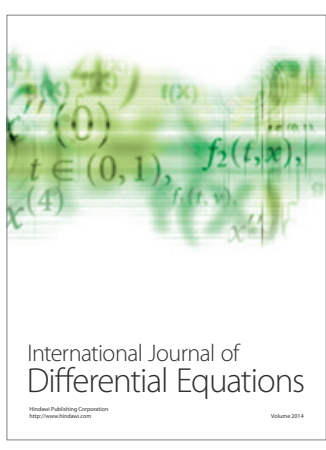
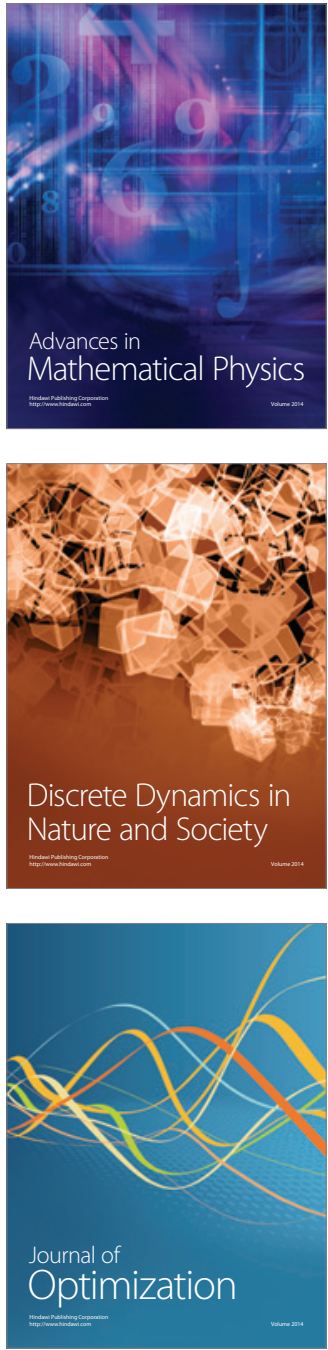